\title{
DNA TRANSISTORS-A THEORETICAL REVIEW
}

\section{MARY JAYA V J}

Department of Computer Science, Assumption College, Changanacherry, India

\begin{abstract}
The ability of a DNA to conduct electrical current was found to be an extraordinary effect of science which made it consider as a molecule which may have much more roles in the nature which was still unknown. DNA transistor is such a kind of technology which considers biological ideas in order to build electronic transistors, which are indeed an immune factor in the development of electronics that could order without human skill. This paper prudently points towards the review on literature of DNA transistors that could overcome that classical silicon based transistor electronics face with the help of nanotechnology by designing specific electrical circuits.
\end{abstract}

KEYWORDS: DNA Transistors, Carbon Nanotubes, Nanotechnology, Bio Molecular Device.

Received: Mar 02, 2019; Accepted: Mar 22, 2019; Published: Jun 25, 2019; Paper Id.: IJMPERDJUN2019197

\section{INTRODUCTION}

Transistors are said to be the building blocks of electronics due to its ability to be switched on and off by applying an electric current to them. We have always been looking forward for tools in order to create nanocircuits which could assemble themselves and thus enables manufacturing of nanoscale electronics in a large scale, and DNA is such a natural molecule to look into to do the same. Even though DNA could self-assemble themselves to work certain tasks and act as a building block, they cannot be considered completely as a good conductor, and to achieve this, strands of DNA can be modified, by adding proteins from certain strains of bacteria to the required segment of DNA molecule and then molecules of protein to test tube along with proteins that are coated with carbon nanotubes.

We can consider a transistor as the basic unit of an integrated circuit engrave into copious film of silicon. During the manufacture of a transistor, for benefitting them as a switch linking a conducting and a non-conducting state, numerous foreign atoms are thrust into it. If an antecedent transistor of DNA were developed with size onetenth when considered to the existing transistors and amalgamated on a chip, then they could have been smaller than the tiniest one that could have been hatched with the surviving technology. A Pentium chip, which is a present-day microprocessor has a surplus of about 4- million transistors as that on a Silicon chip which is only more or less larger than a thumbnail. [1]. Information in a computer chip is processed by a union of billions of tiny transistors. Efficiency and speed of these transistors depend on their size, smaller the size, larger the amount that can be embedded into a chip, so greater is the speed and efficiency. Over a speck of years, there have been going extensive research works in order to build minute transistors in nanometre scale.

The idea of developing nano-logical devices from DNA molecules were from the attempt of improvising new smaller and faster logical devices. It is known that the di-phosphide bridges present in the DNA molecule can act as tunnel junctions in the coulomb blockage regime, and the Hydrogen bonds have properties of capacitance and, all these are theoretically predicted knowledge. All these devices are made entirely from DNA, where what required externally is just sources and loads. The logical devices developed from DNA molecules would be in the 
nanometre scale and is possible to work at room temperature. What makes them extremely fast is just their operation principle. All these devices are made from certain specific molecules which makes them completely identical to each other, and due to the presence of highly stabilised chemical bonds, they would also be highly stable. The property of DNA molecules, which is the ability to self-assemble themselves, they are used to create convoluted networks consisting of many elements [2].

In this paper, we shall discuss the technical ability to fleece a DNA strand with different metals, so as to form a conductive wire with self- assembling properties as that of the DNA molecule. Section II and III describes DNA electronics and conducting nature of DNA. Section IV discuss how a DNA transistor can be constructed. Section V lists advantages and application. Finally, Section VI concludes the discussion.

\section{DNA ELECTRONICS}

Basically a single strand of DNA built up by units comprising of a sugar and a base. The kernels are connected by different Phosphorous bridges or else what we call the P-Bonds, whereas the complementary bases in different strands are connected by hydrogen bonds or H-Bonds [3]. The P-bonds provides with the net charge in order to for a tunnel junction. The coupling to the environmental degrees of freedom determines either the tunnelling is stochastic or coherent [4]. When two oxygen atoms diagonally connected to the phosphorus atom, it is what we call as the origin of this tunnel junction. Now, two $\sigma$ bonds and one $\pi$ bonds are made up by the oxygen, when it shares three electrons with the phosphorous. As the lowest level is occupied by $\pi$ electron, it resembles an electron in a double well potential [5]. When an additional electron proceeds towards the well, a narrow barrier is encountered, due to the presence of an energy gap to the next level of the well and these electrons can easily tunnel through it. The proton present in the H-bond can produce a net charge density on either side of the bond due to its capacitive properties by just shifting its position [2]. All these kernels themselves have properties of inductance, developed from the hopping of excessive electrons.

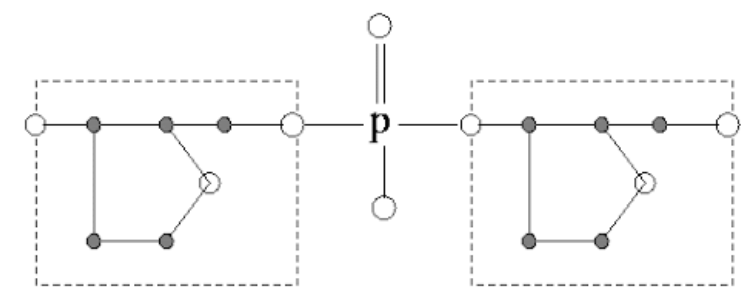

Figure 1: Two Grains in this Distinction DNA Connected by a P-Bond.

Figure 2 shows the schematic image of two kernels in DNA connected by a P-bond. The dark circles represents carbon atoms and the white circles oxygen atoms. 


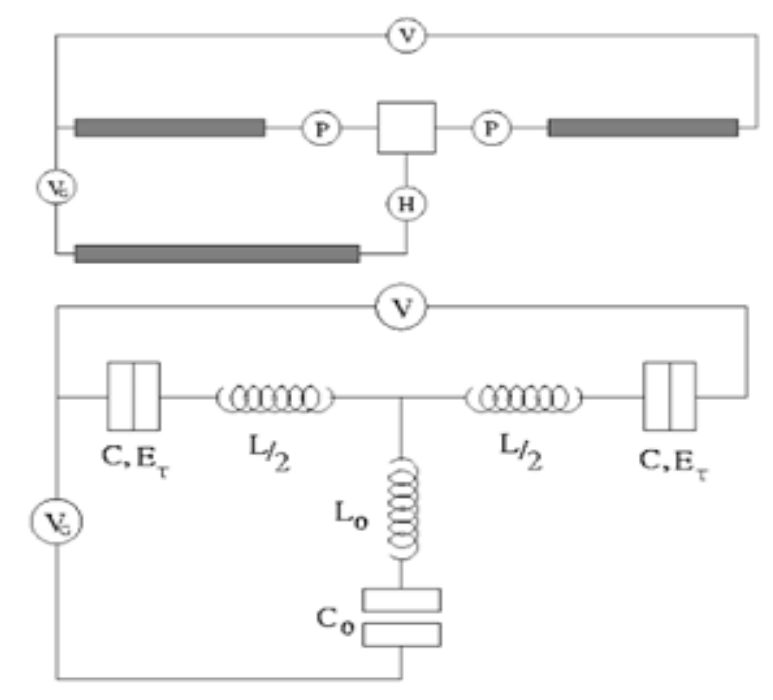

Figure 2: A Schematic Image of DNA set (above) and Equivalent Circuit (below)

Figure 2 shows that the DNA molecule exhibits all the properties needed for logical devices. P and $\mathrm{H}$ denotes the P-bonds between the sugars, and H-bond between the bases respectively. $\mathrm{V}$ and $\mathrm{V}_{\mathrm{G}}$ are the external and the gate voltages. $\mathrm{C}$ is the capacitance and $\mathrm{E}_{\mathrm{r}}$ is the tunnelling energy of the P-bond. $\mathrm{C}_{\mathrm{O}}$ is the capacitance of the H-bond and $\mathrm{L}$ and $\mathrm{L}_{\mathrm{o}}$ are the longitudinal and the lateral inductances respectively [6].

\section{CONDUCTING NATURE OF DNA}

Normally DNA is either an insulator or a semiconductor. A wide bandgap semiconductor using DNA and other polymers can be developed, by applying a voltage across them and successively inducing charge transport through it without changing the structure [7]. If the structure is permanently damaged or changed it permanently becomes an insulator.

Fink and Schonenberger were the first to measure the current flow through a DNA using a modified low-energy electron point-source microscope at room temperature. This technique was developed by using a gold-coated carbon grid, consisting of an array of 2- $\mu \mathrm{m}$ holes and 600-nm-long DNA to span these holes. A tip manipulated with tungsten was used to make a contact with the sample and to allow electric current to flow. The measurement of linear current voltage were done by curves in the range of -20 to $+20 \mathrm{mV}$, and a resistance of $2.5 \mathrm{M}$ per DNA molecule [8]. The fact that DNA act as a molecular wire is confirmed by various other studies, by the role of tunnel junctions done by the phosphate bonds in the DNA for electrons to move along. It was possible for a Dutch team to show that a $10.4 \mathrm{~nm}$ long (30 base pair) poly (G)poly(C) sequence has electrical characteristics which were similar to that of a semi conducting diode that allows current to flow in only a single direction. The measurement of $\mathrm{I} / \mathrm{V}$ curves were in the range of -4 to $+4 \mathrm{~V}$ and found that the measured current flow did not fall more than $1 \mathrm{pA}$ below a certain threshold value. The DNA behaved as an insulator in the given voltage range. The current increased sharply, beyond this threshold voltage, which shows that the DNA conducts charge and thus behaves in a similar way as that of a silicon based semiconductor.

Researchers even have reported that DNA can behave sort of a proximity induced superconductor, using $16 \mu \mathrm{m}$ of DNA to attach two rhenium carbon electrodes on a mica substrate. The conductivity of DNA will be increased by coating it with metals in various ways. Aich and colleagues went one step further and showed that DNA with zinc atoms incorporated between its bases also acts as a conductor. Other metals, like nickel and cobalt can similarly be used. On the 
premise of those results, DNA has the potential to self-assemble to make any circuit and to function as a template for the deposition of metal atoms.

The electrical properties of DNA might get modified due to various alterations to its material properties, such as its sequence, diameter and stiffness. While exploring the potential of DNA as an electric wire, it was found that guanine is the base with the lowest oxidation potential. It loses an electron during oxidative stress and becomes positively charged. This positive charge does not stay at the base where it was formed, but keeps moving along G-rich sequences, which lowers the molecules oxidative potential. Thus a positive charge moves from a single guanine towards a multiple guanine sequence thereby attracting electron holes.

$(\mathrm{G}+\mathrm{C})$ rich DNA shows p-type properties and $(\mathrm{A}+\mathrm{T})$ sequences show n-type ones. Combining such DNA molecules could create logic elements that would be more powerful than any silicon-based device. Theoretically, just a short sequence of DNA base pairs may be enough to create all the combined $\mathrm{n}$ and $\mathrm{p}$ type properties [8]. Electrons from the negative pole, beyond a certain breakthrough voltage, jump through the island over the adenine/ thymine barriers to allow electrons flow to the positive pole.

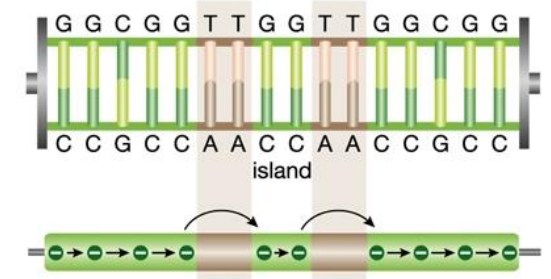

Figure 3: A Hypothetical Resonant Tunnelling Diode Based on DNA

Figure 4 shows a hypothetical resonant tunnelling diode supported DNA. It consists of guanine/ cytosine rich donor and acceptor sequences at the poles, which are interrupted by two adenine/ thymine rich sequences and short island of guanine and cytosine. In silicon based resonant tunnelling diode the barriers between the conducting parts stop current from flowing. Above a particular voltage, electrons can pass over the tiny island and current begins to flow. In a DNA based resonant tunnelling diode, the guanine/ cytosine sequences would accept.

\section{BUILDING A DNA TRANSISTOR}

Let us consider how a classical SET transistor is built. First, start with two strands (a main strand and a gate strand), and connect the end base of the gate strand to a complementary base in the middle of the main strand. Both strands should be metal coated, except the grain within the middle strand which is connected to the gate strand, and its two adjacent P-bonds. The connective H-bond should be uncoated. The coated and un-coated parts are composed of specific, yet different sequences of bases for feasibility. Before the coating, the DNA molecule should be in solution containing an enzyme which can bound only to the parts which should not be coated. After the coating the enzyme is released, and desired result is obtained. The metallic coated ends of the most strand will be now connected to a voltage source $\mathrm{V}$ and also the end of the gate strand to a different voltage source, $\mathrm{V}_{\mathrm{G}}$ which acts as a gate voltage.

This DNA made device has the structure of a SET transistor, i.e., a grain connected by two tunnel junctions to a voltage source, and biased by a capacitive coupling to a gate voltage. The inductive properties of the grain may sometimes be neglected, which is equivalent to the claim that the relaxation time in the grain is much shorter than the tunnelling time. The values of $\mathrm{L}$ and $\mathrm{L}_{\mathrm{O}}$ in the DNA molecule are not known, but should be very small, probably in the pico-henry range. 
Thus the relaxation time is very short, and the inductances can be neglected. Also assume that the coupling to the environment is strong such that the tunnelling is incoherent and the discrete energy levels in the grain are smeared. In this case the voltages across the two junctions are

$$
\begin{gathered}
V_{1}=V \frac{C_{2}}{C_{1}+C_{2}}-\frac{N_{e}+V_{G} C_{0}}{C_{1}+C_{2}} \\
V_{2}=V \frac{C_{1}}{C_{1}+C_{2}}-\frac{N_{e}+V_{G} C_{0}}{C_{1}+C_{2}} \quad-\rightarrow \text { (1) }
\end{gathered}
$$

Where $\mathrm{N}$ is the number of surplus electrons in the grain, and e is the electron charge. Suffixes were added to distinguish between the two tunnel junctions. The instantaneous rate of tunnelling from the right across the first junction is calculated from the single electron energy levels.

$$
\mathrm{C}=\int_{-x}^{x} \frac{2 \pi}{h}[T(E)]^{2} D_{g r}\left(E-E_{g r}\right) D_{r}\left(E-E_{r}\right) X f\left(E-E_{r}\right)\left[1-f\left(E-E_{g r}\right)\right] d E--\rightarrow(2)
$$

Where $T(E)$ is the tunnelling matrix element for an electron in a state of energy $E, f(E)$ is the Fermi distribution function, $\mathrm{D}_{\mathrm{gr}}(\mathrm{E})$ and $\mathrm{D}_{\mathrm{r}}(\mathrm{E})$ are the density of states for the grain and the right electrodes respectively and similarly $\mathrm{E}_{\mathrm{gr}}$ and

$E_{r}$ are their highest occupied energies. Their difference gives rise to a coloumb blockade of tunnelling into the grain. The system obeys the Fermi distribution due to the large coupling to the environment. The other tunnelling rates $r_{2}\left(V_{2}\right), l_{1}\left(V_{1}\right)$, and $l_{2}\left(V_{2}\right)$ have similar expressions. The probability that there are $\mathrm{N}$ electrons in the grain at time $t$ is governed by the master equation [9]

$$
\begin{aligned}
& \frac{\partial p(N, t)}{\partial t}=\left[r_{1}(N-1)+l_{2}(N-1)\right] \rho(N-1, t)+\left[l_{1}(N+1)+r_{2}(N+1)\right] x \rho(N+1, t) \\
& \quad-\left[r_{1}(N)+l_{1}(N)+r_{2}(N)+l_{2}(N)\right] \rho(N, t)---\rightarrow(3)
\end{aligned}
$$

Where the transition rates are expressed as functions of $\mathrm{N}$ using equation (1). The I-V characteristics of the SET can be obtained by solving equation (3) numerically using the appropriate initial and boundary conditions. A typical I-V characteristic for constant density of energy states and identical junctions in the low temperature limit has a voltage threshold. In order to operate as a transistor, $\mathrm{V}_{\mathrm{G}}$ is varied around the threshold voltage. For well-functioning transistor characteristics, the current that raise above the threshold value must be kept as steep as possible [10]. This can be achieved if the tunnelling rates of the two junctions are different or if there is a gap in the density of energy states of the grain.

As DNA molecule is not conductive it possess a natural energy gap. This can be enhanced by using a larger section of DNA containing several grains instead of a single one. This happens since long DNA chain have non-linear effects, resulting in the tendency of charges to form solution.

\section{ADVANTAGES AND APPLICATION}

DNA Transistors are very small in size. So implementing these transistors in IC would reduce the chip area and so more number of chips can be fabricated on devices like computers and sensors. Thus much faster device can be constructed.

The realization of individual molecules or nano-scaled units as functional devices would be a good medicine for the limitation of an electric circuit technology. These transistors may enable the creation of any number of devices in future 
applications. Computers are only one application. It is also hoped that these transistors would be able to build a platform for next generation bio-intelligent medicine. They can have a wide range of market application in biosensors, bioengineering, medical diagnostics, medical devices and bio-informatic chip platform.

\section{CONCLUSIONS}

DNA Transistor is an emerging technology. The commercialization of a small chi made with DNA transistors may take years of research. The applications are limited only by our imagination. We could one day have living bridges or buildings constructed by the self-directed growth of their natural materials, controlled by internal biological circuits. DNA transistors are thus outstanding research in the area that matters most in nanotechnology.

\section{ACKNOWLEDGEMENT}

I thank each and every individual who has supported me to get a literature review on this topic successfully. I express my sincere thanks to my colleagues for their kind help.

\section{REFERENCES}

1. BHALLA, Vijayender, Ram P. BAJPAI, and Lalit M. BHARADWAJ. "DNA electronics," EMBO reports, vol. 4, no. 5, pp. 442445, 2003.

2. Ben-Jacob, E., Z. Hermon, and S. Caspi. "DNA transistor and quantum bit element: realization of nano-biomolecular logical devices," Physics Letters A vol. 263, no. 3, pp. 199-202, 1999.

3. Knotts IV, T. A., Rathore, N., Schwartz, D. C., \& De Pablo, J. J “A coarse grain model for DNA," The 02B611, 2007.

4. Paladino, E., Galperin, Y.M., Falci, G. and Altshuler, B.L., "Iff noise: Implications for solid-state quantum information," Reviews of Modern Physics, vol. 86, no. 02, pp. 361, 2014.

5. Song, H., Reed, M.A. and Lee, T., "Single molecule electronic devices" Advanced Materials vol. 23, no. 14, pp. 1583-1608, 2011 .

6. Mozetič, M., Vesel, A., Primc, G., Eisenmenger-Sittner, C., Bauer, J., Eder, A., Schmid, G.H., Ruzic, D.N., Ahmed, Z., Barker, D. and Douglass, K.O, "Recent developments in surface science and engineering, thin films, nanoscience, biomaterials, plasma science, and vacuum technology," Thin solid films, volume 660, pp. 120-160, 2018.

7. Pearton, S.J., Ren, F., Wang, Y.L., Chu, B.H., Chen, K.H., Chang, C.Y., Lim, W., Lin, J. and Norton, D.P.," Recent advances in wide bandgap semiconductor biological and gas sensors", Progress in Materials Science, volume 55, no 01, pp. 1-59, 2010.

8. Bhalla, V., Bajpai, R.P. and Bharadwaj, L.M., "DNA electronics," EMBO reports, vol. 04, no. 05, pp. 442-445, 2003.

9. Hanke, U., Galperin, Y.M., Chao, K.A. and Zou, N., "Finite-frequency shot noise in a correlated tunneling current," Physical Review B, vol. 48, no. 23, pp. 7209, 1993.

10. Schwierz, F., "Graphene transistors", Nature nanotechnology, vol. 05, no. 07, pp. 487, 2010.

\section{AUTHORS PROFILE}




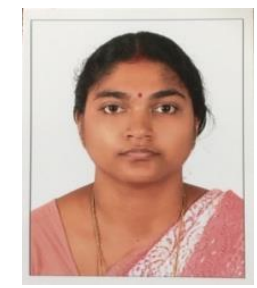

Mary Jaya V J Assistant Professor in Electronics, Department of Computer Science, Assumption College, Changanacherry. Doing Research in medical image processing at Mahatma Gandhi University Regional Centre, Edappally, Kochi-24, Kerala, India. Qualified M.Tech (Electronics) with specialization in Digital Electronics in 2005 from Cochin University of Science and Technology. Qualified M.Sc (Electronics) with specialization in Computer Technology in 2001 from Cochin University of Science and Technology. Areas of interests include image processing, neural networks. 
